\title{
Interleukin-17 Alteration in First-Episode Psychosis: A Meta-Analysis
}

\author{
Xinyu Fang ${ }^{a}$ Yi Zhang ${ }^{a}$ Weixing Fan ${ }^{b}$ Wei Tang ${ }^{c}$ Chen Zhang ${ }^{a}$ \\ ${ }^{a}$ Schizophrenia Program, Shanghai Mental Health Center, Shanghai Jiao Tong University School of \\ Medicine, Shanghai, ${ }^{\text {b}}$ Department of Psychiatry, Jinhua Second Hospital, Jinhua, and ${ }^{~}$ Department of \\ Psychiatry, Wenzhou Kangning Hospital, Wenzhou Medical University, Wenzhou, PR China
}

\section{Keywords}

First-episode psychosis · Schizophrenia · Interleukin-17 . Drug-naïve patients $\cdot$ Immune system $\cdot$ Inflammatory processes

\begin{abstract}
Schizophrenia is accompanied with central nervous system and peripheral immune system imbalances. Interleukin-17 (IL-17) is implicated in various immune and inflammatory processes. Aberrant levels of IL-17 have been reported in patients with schizophrenia, whereas the results are not consistent. To clarify the relationship between IL-17 and schizophrenia, we performed a meta-analysis in this study. We carried out a structured literature search in PubMed and Embase database up to April 16, 2017, and retrieved all eligible casecontrol studies according to the inclusion criteria. Finally, a total of 313 patients with drug-naïve first-episode psychosis and 238 healthy control subjects from 5 studies were included in our meta-analysis. There were no significant differences between first-episode psychosis patients and healthy controls with respect to the levels of IL-17 ( $p=0.21$ ), even when we removed 2 studies which were not European samples ( $p=0.12$ ). Our findings suggested that IL-17 may not be involved in the pathological mechanism of schizophrenia.
\end{abstract}

(c) 2017 S. Karger AG, Basel (c) 2017 S. Karger AG, Basel

\section{Introduction}

Schizophrenia is a chronic and debilitating mental disorder with a prevalence rate of approximately $1 \%$ of the worldwide population [1]. Although clinical symptomatology of this disorder is diverse, psychosis is the core symptom, which includes positive, negative, and cognitive symptoms [2]. Early literature has documented that schizophrenia is mainly caused by the interaction of hereditary and environmental factors [3]. However, its genuine biological mechanism is still unknown. Over the past decades, abnormal immune-inflammatory responses, especially aberrant levels of pro-inflammatory cytokines, have been found in patients with schizophrenia $[4,5]$ and their relatives [6]. Furthermore, accumulating evidence has shown that immune-related genes confer susceptibility to schizophrenia $[7,8]$. Therefore, immune abnormalities may play an important role in the pathophysiology of schizophrenia.

Cytokine is one of the most important components in the immune system and the principal cells that can synthesize and release cytokines are CD4+ T lymphocytes. Peripheral cytokines can penetrate the blood brain barrier and bind to receptors on glial and neuron cells in the brain [9]. Th17 cell emerged as a new CD4+ cell subset, which infiltrates the central nervous system through the

\section{KARGER}

E-Mail karger@karger.com www.karger.com/mnp 
direct effects of its cytokine production [10]. Interleukin-17 (IL-17) is the product of Th17 cells and implicated in various immune and inflammatory processes [11], and there exists evidence supporting the association of IL-17 with schizophrenia $[12,13]$. It is being increasingly recognized that gut permeability may play a pivotal role in schizophrenia [14]. Given that Th17 cells play a predominant role in mucosal cell integrity and defense [15], Th17 cells and their major product IL-17 may intimately interact with schizophrenia. In animal models, maternal immune activation strongly predisposes offspring to schizophrenia risk [16]. It is demonstrated that prenatal infection leads to the development of Th17 cells and might affect the production of IL-17 in mice offspring [17]. All of these pieces of evidence suggest that IL-17 dysfunction may be involved in the immune abnormalities of schizophrenia.

Although recent studies reported increased IL-17 concentrations in antipsychotic-naïve psychotic patients [13, 18], the results were not consistent [19-21]. Such poor replication can arise from several different factors. For example, small sample sizes result in low statistical power of detection, coinciding with increased rates of both false positives and false negatives and subsequently difficulty in replicating or refuting previous results. Overcoming these limitations is difficult; however, meta-analysis offers a superior option since it allows easily surveying a broad set of subjects, thereby enhancing power and allowing for a more broadly based analysis of previously available data.

To the best of our knowledge, there is no meta-analysis exploring the association between IL-17 and psychosis. To address the conflicting results, we pooled results of all published original association studies to determine the overall strength of a putative association between IL-17 and schizophrenia. It is known that IL-17 expression is influenced by antipsychotic drugs. Therefore, we only included drug-naïve patients in this study.

\section{Methods}

\section{Study Selection}

We systematically searched through computerized literature databases PubMed and Embase before April 16, 2017. The primary search strategy was (schizophrenia or psychosis or schizophrenic disorder) and (interleukin-17 or interleukin 17 or IL-17 or IL17 or IL 17), limiting the results to human studies published in English. The reference lists of identified articles were also screened. First, all studies were imported to the literature management software Endnote X7 to eliminate duplicated records. Then we conducted a preliminary screening of reports by reading titles and ab- stracts. Some initial matches were excluded because they (1) were review articles, (2) were animal studies, (3) were genetic studies related to cytokines, (4) were in vitro studies, or (5) did not present cytokine data. Finally, the full texts of potentially relevant articles were downloaded for the second round of screening. The inclusion criteria were as follows: (1) published in English before April 16, 2017, (2) patients with first-episode psychosis (FEP) including the following schizophrenia spectrum disorder: schizophrenia, schizoaffective disorder, schizophreniform disorder, and psychotic disorders not otherwise specified, (3) diagnosis was established using International Statistical Classification of Diseases and Related Health Problems (ICD) or Diagnostic and Statistical Manual of Mental Disorders (DSM), (4) assessment of circulating cytokine IL-17 levels with blood samples (in vivo studies), and the blood samples from the patients were collected before application of antipsychotics, and (5) data was presented as mean and standard deviations (SD) (or standard error, SE) for IL-17 about patients and healthy controls. The exclusion criteria were: (1) studies without a control group, (2) studies that did not present mean and SD (or SE) for IL-17 level, and (3) patients with FEP having received antipsychotic drug treatment.

Two authors independently completed the preliminary screening based on titles and abstracts and retrieved full texts to evaluate final eligibility of studies according to relevant inclusion criteria. Any discrepancies were resolved by discussion and consensus.

\section{Data Extraction and Statistical Analysis}

We extracted data from identified studies including the name of the first author, year of publication, location of the study, diagnosis of FEP, and criteria used to make the diagnosis. Publications pertaining to the same research group were checked for potential data overlap. Sample size, mean, and SD for drug-naïve FEP and control subjects for IL-17 assessed in each study were recorded. Results were converted to standard units $(\mathrm{pg} / \mathrm{mL})$ when necessary, and the SE [19] was transformed to SD through the formula: $\mathrm{SD}=\mathrm{SE} \times \sqrt{ } n$ ( $n$ : sample size). After recording this information, data were pooled into a database and analyzed using Review Manager 5.3 software. The random-effects model was selected as more conservative than the fixed-effects model, giving a lower type I error and wider confidence intervals. Its usage was indicated by the considerable heterogeneity in the studies included [22].

\section{Results}

\section{Study Characteristics}

A total of 95 articles were retrieved. After removing duplicated records and screening the data according to the predefined inclusion and exclusion criteria, 9 studies were selected. Among the 9 articles, 2 studies by Borovcanin and colleagues published in 2012 [19] and 2013 [23] were based on the same sample, and 2 studies by Noto and colleagues published in 2014 [24] and 2015 [21] were also based on the same sample. Additionally, 2 studies included a mixture of samples of drug-naïve pa- 
Fig. 1. Flowchart of included/excluded studies. SD, standard deviation; FEP, firstepisode psychosis. $n=95$ records identified through database

searching (PubMed $=25$, Embase $=70$ )

Adjusting for duplicates

$n=70$ records screened

Excluded after title and

abstract screened $(n=36)$

$n=34$ full-text articles assessed for eligibility

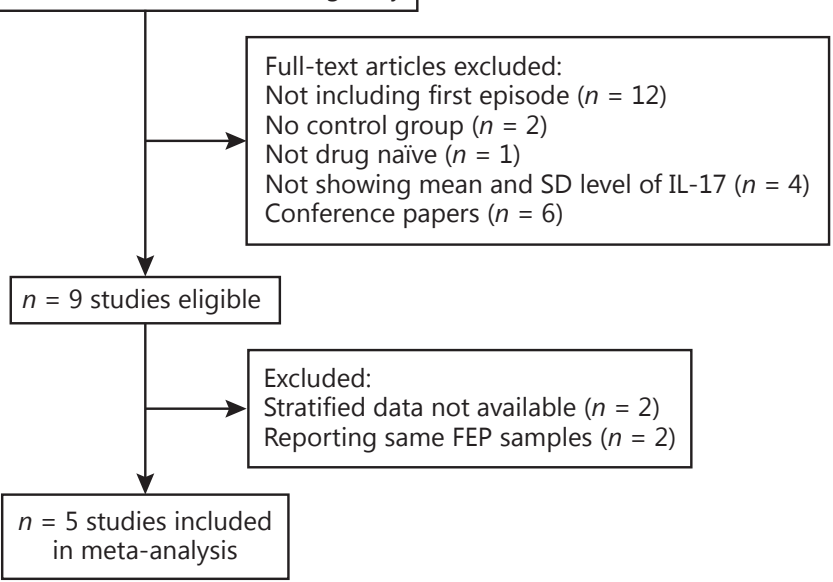

Table 1. Characteristics of the 5 included studies

\begin{tabular}{|c|c|c|c|c|c|c|c|c|}
\hline Study & Country & Ethnicity & $\begin{array}{l}\text { Diagnostic } \\
\text { criteria }\end{array}$ & Tissue & $\begin{array}{l}\mathrm{FEP}, n \\
(\text { mean } \pm \mathrm{SD})\end{array}$ & $\begin{array}{l}\mathrm{HC}, n \\
(\text { mean } \pm \mathrm{SD})\end{array}$ & $\begin{array}{l}\text { Age } \\
\text { range, } \\
\text { years }\end{array}$ & Assay \\
\hline Borovcanin et al. [19] (2012) & Serbia & European & ICD-10 & serum & $88(6.57 \pm 43.34)$ & $36(30.86 \pm 83.7)$ & $\mathrm{N} / \mathrm{A}$ & ELISA \\
\hline Ding et al. [13] (2014) & China & Asian & DSM-4 & plasma & $69(17.69 \pm 6.37)$ & $60(15.61 \pm 5.46)$ & $18-45$ & ELISA \\
\hline Schwarz et al. [18] (2012) & Germany & European & DSM-4 & serum & $71(14.48 \pm 7.85)$ & $59(9.42 \pm 3.36)$ & $\mathrm{N} / \mathrm{A}$ & Human MAP \\
\hline Noto et al. [21] (2015) & Brazil & Latin American & DSM-4 & serum & $55(2.31 \pm 4.76)$ & $57(4.26 \pm 11.87)$ & $16-40$ & CBA \\
\hline Şimşek et al. [20] (2016) & Turkey & European & DSM-5 & serum & $30(7.6 \pm 1.62)$ & $26(1.9 \pm 6.8)$ & $10-17$ & $\mathrm{CBA}$ \\
\hline
\end{tabular}

FEP, first-episode psychosis patients; HC, healthy controls; SD, standard deviation; N/A, not applicable.

tients and those drug free for at least 3 months $[12,25]$. Because no stratified data was provided in the articles, we excluded these studies. Finally, 5 articles (a total of 313 patients with drug-naïve FEP and 238 healthy control subjects) were included in this meta-analysis (Fig. 1) [13, 18-21]. With regard to the tissue of the blood samples, all of them were from serum except for one from plasma [13] (Table 1).

Interleukin-17 Alteration in First-Episode Psychosis

\section{Meta-Analysis}

As shown in Figure 2, there were no significant differences in blood levels of IL-17 $(Z=1.25, p=0.21)$ between FEP and HC subjects, while there was a high heterogeneity in effect size estimates $\left(I^{2}=77 \%\right)$. In subgroup analyses, although the heterogeneity declined $\left(I^{2}=50 \%\right)$, we still did not find statistical differences after removing 2 studies which were not European samples $(Z=1.57, p=$ $0.12)[13,20]$. 


\begin{tabular}{|c|c|c|c|c|c|c|c|c|c|c|}
\hline \multirow[t]{2}{*}{ Study or subgroup } & \multicolumn{3}{|l|}{ FEP } & \multicolumn{3}{|l|}{$\mathrm{HC}$} & \multirow{2}{*}{$\begin{array}{l}\text { Weight, } \\
\%\end{array}$} & \multirow{2}{*}{$\begin{array}{l}\text { Mean difference } \\
\text { IV, random }(95 \% \mathrm{CI})\end{array}$} & \multirow{2}{*}{\multicolumn{2}{|c|}{$\begin{array}{l}\text { Mean difference } \\
\text { IV, random, 95\% CI }\end{array}$}} \\
\hline & mean & SD & total & mean & SD & total & & & & \\
\hline \multicolumn{11}{|c|}{ Comparison in a mixed population } \\
\hline Borovcanin et al. [19] & 6.57 & 43.34 & 88 & 30.86 & 83.7 & 36 & 1.3 & $-24.29(-53.09,4.51)$ & $\leftarrow$ & \\
\hline Ding et al. [13] & 17.69 & 6.73 & 69 & 15.61 & 5.46 & 60 & 29.2 & $2.08(-0.02,4.18)$ & & $\rightarrow-$ \\
\hline Şimşek et al. [20] & 7.6 & 16.2 & 30 & 1.9 & 6.8 & 26 & 15.2 & $5.70(-0.66,12.06)$ & & \\
\hline Subtotal $(95 \% \mathrm{CI})$ & & & 313 & & & 238 & 100.0 & $-2.15(-1.22,5.53)$ & & \\
\hline \multicolumn{11}{|c|}{$\begin{array}{l}\text { Heterogeneity: } \tau^{2}=9.03 ; \chi^{2}=17.29, \mathrm{df}=4(p=0.002) ; P^{2}=77 \% \\
\text { Test for overall effect: } Z=1.25(p=0.21)\end{array}$} \\
\hline Subtotal $(95 \% \mathrm{CI})$ & & & 189 & & & 121 & 100.0 & $-4.31(-1.08,9.70)$ & & \\
\hline \multicolumn{11}{|c|}{ Heterogeneity: $\tau^{2}=11.17 ; \chi^{2}=4.02, \mathrm{df}=2(p=0.13) ; R^{2}=50 \%$} \\
\hline \multicolumn{9}{|c|}{ Test for overall effect: $Z=1.57(p=0.12)$} & $-10-5$ & $\begin{array}{lll}1 & 5 & 10\end{array}$ \\
\hline \multicolumn{9}{|c|}{ Test for subgroup differences: $\chi^{2}=0.44, \mathrm{df}=1(p=0.51) ; l^{2}=0 \%$} & \multicolumn{2}{|c|}{ Favors HC Favors FEP } \\
\hline
\end{tabular}

Fig. 2. Random-effects meta-analysis of serum level of IL-17 in drug-naïve first-episode psychosis patients (FEP) versus healthy controls (HC). Mean and standard deviation (SD) are shown in $\mathrm{pg} / \mathrm{mL}$.

\section{Discussion}

This is the first meta-analysis addressing the blood IL17 levels in drug-naïve FEP patients and normal healthy controls. The major finding of our meta-analysis is that blood IL-17 levels have no significant differences between these two groups in a mixed population. After removing 2 studies from Asian and Latin America, we also did not find statistical differences between the case and control groups. Our results implied that IL-17 may not be involved in the pathological mechanism of schizophrenia; however, our results should be interpreted with caution in light of small numbers of studies and high heterogeneity and some potential confounding factors.

The main strength of this meta-analysis is that we strictly examined drug-naïve FEP patients, whereas previous meta-analyses often included a mixture of drug-naïve and drug-free populations, ruling out the compacts of some confounding factors which can interfere with immunological processes of the human body, i.e., administration of antipsychotics and the course of disease. Ding et al. [13] found a significant decrease in the proportion of Th17 cells and a trend of decrease in plasma levels of
IL-17 after 4 weeks of risperidone treatment in first-episode schizophrenia, and Dimitrov et al. [26] found significantly decreased levels of IL-17 in chronic schizophrenia patients compared to the controls. In contrast, Himmerich et al. [27] demonstrated that antipsychotics may increase the production of IL-17. Taken together, antipsychotic treatment may have a direct impact on the levels of IL-17 and confuse the results of IL-17 in psychosis. It has been proposed that chronically activated immune cells and other related cells release inflammatory cytokines that give rise to the structural and functional neuronal changes over time seen in schizophrenia [28]. Meanwhile, chronic cytokine secretion may be caused by the out-of-balance state of the neurotransmitter such as dopamine and gamma-amino butyric acid (GABA- $\gamma$ ) in schizophrenia [29]. Therefore, studies performed in drug-naïve FEP patients might provide stronger evidence to understand the role of inflammation in psychiatric diseases.

There are several limitations in this study. First, we only included IL-17 in this study, and it may result in potential bias. Second, there is a high heterogeneity of included studies in this meta-analysis. This may be caused 
by the samples that originate from different areas. Third, it should be noted that most potential confounding factors were not fully controlled in our included studies, such as age, smoking habits, body mass index, etc. Finally, it is known that the time of sampling and whether blood was taken under fasting conditions may influence the level of IL-17. Although all of the blood samples were obtained before the administration of the first dose of antipsychotics, most studies did not point out the sampling time and whether there was fasting or not.

In summary, further investigations need to focus on the drug-naïve FEP patients at the early stages and in numbers large enough to allow for other confounding factors known to influence cytokine levels to be controlled, and to explore IL-17 level in brain tissues, not just peripheral level, in order to provide robust evidence regarding the role of IL-17 in the immune-inflammatory mechanisms of psychotic disorders. More importantly, rather than single cytokines, studies should to explore more related molecules in a common pathway. To ex- plore the interaction of cytokines, hormones, and neurotransmitters in larger studies of higher quality may lead to further improvement for diagnosis of schizophrenia and other specific psychotic diseases and to finding new strategies for better treatment approaches.

\section{Acknowledgements}

This work was supported by the National Natural Science Foundation of China (81471358 and 81771450), the Shanghai Science and Technology Commission Foundation (14411969000), the Shanghai Municipal Education Commission - Gaofeng Clinical Medicine Grant Support (20152530), the Shanghai Municipal Commission of Health and Family Planning Foundation (201540029), and the Shanghai Mental Health Center Foundation (2014-FX-03).

\section{Disclosure Statement}

There are no conflicts of interest to report.

\section{References}

1 Insel TR: Rethinking schizophrenia. Nature 2012;468:187-193.

2 Zhang C, Li Z, Shao Y, Xie B, Du Y, Fang Y, Yu S: Association study of tryptophan hydroxylase-2 gene in schizophrenia and its clinical features in Chinese Han population. J Mol Neurosci 2011;43:406-411.

3 Cannon TD, van Erp TG, Bearden CE, Loewy R, Thompson P, Toga AW, Huttunen MO, Keshavan MS, Seidman LJ, Tsuang MT: Early and late neurodevelopmental influences in the prodrome to schizophrenia: contributions of genes, environment, and their interactions. Schizophr Bull 2003;29:653-669.

4 Fu YY, Zhang T, Xiu MH, Tang W, Han M, Yun LT, Chen DC, Chen S, Tan SP, Soares JC, Tang WJ and Zhang XY: Altered serum levels of interleukin-3 in first-episode drug-naive and chronic medicated schizophrenia. Schizophr Res 2016;176:196-200.

5 Zhang XY, Zhou DF, Zhang PY, Wu GY, Cao LY, Shen YC: Elevated interleukin-2, interleukin- 6 and interleukin- 8 serum levels in neuroleptic-free schizophrenia: association with psychopathology. Schizophr Res 2002;57: 247-258.

6 Martinez-Gras I, Garcia-Sanchez F, Guaza C, Rodriguez-Jimenez R, Andres-Esteban E, Palomo T, Rubio G, Borrell J: Altered immune function in unaffected first-degree biological relatives of schizophrenia patients. Psychiatry Res 2012;200:1022-1025.
7 Purcell SM, Wray NR, Stone JL, Visscher PM, O'Donovan MC, Sullivan PF, Sklar P: Common polygenic variation contributes to risk of schizophrenia and bipolar disorder. Nature 2009; 460:748-752.

8 Carey CE, Agrawal A, Bucholz KK, Hartz SM, Lynskey MT, Nelson EC, Bierut LJ, Bogdan R: Associations between polygenic risk for psychiatric disorders and substance involvement. Front Genet 2016;7:149.

9 Yarlagadda A, Hampe CS and Clayton AH: The blood brain barrier and the role of ratiometric molecular analysis in schizophrenia. Psychiatry (Edgmont) 2010;7:20-23.

10 Debnath M, Berk M: Th17 pathway-mediated immunopathogenesis of schizophrenia: mechanisms and implications. Schizophr Bull 2014;40:1412-1421.

11 Zhu S, Qian: IL-17/IL-17 receptor system in autoimmune disease: mechanisms and therapeutic potential. Clin Sci (Lond) 2012;122: 487-511.

12 El Kissi Y, Samoud S, Mtiraoui A, Letaief L, Hannachi N, Ayachi M, Ali BBH, Boukadida $\mathrm{J}$ : Increased Interleukin-17 and decreased BAFF serum levels in drug-free acute schizophrenia. Psychiatry Research 2015;225:58-63.

13 Ding M, Song X, Zhao J, Gao J, Li X, Yang G, Wang X, Harrington A, Fan X, Lv L: Activation of Th17 cells in drug naïve, first episode schizophrenia. Prog Neuropsychopharmacol Biol Psychiatry 2014;51:78-82.
14 Severance EG, Prandovszky E, Castiglione J, Yolken RH: Gastroenterology issues in schizophrenia: why the gut matters. Curr Psychiatry Rep 2015;17:27.

15 Abraham C, Cho J: Interleukin-23/Th17 pathways and inflammatory bowel disease. Inflamm Bowel Dis 2009;15:1090-1100.

16 Brown AS: Epidemiologic studies of exposure to prenatal infection and risk of schizophrenia and autism. Dev Neurobiol 2012;72:12721276.

17 Mandal M, Marzouk AC, Donnelly R, Ponzio NM: Preferential development of Th17 cells in offspring of immunostimulated pregnant mice. J Reprod Immunol 2010;87:97-100.

18 Schwarz E, Guest PC, Rahmoune H, Harris LW, Wang L, Leweke FM, Rothermundt M, Bogerts B, Koethe D, Kranaster L, Ohrmann P, Suslow T, McAllister G, Spain M, Barnes A, Van Beveren NIM, Baron-Cohen S, Steiner J, Torrey FE, Yolken RH, Bahn S: Identification of a biological signature for schizophrenia in serum. Mol Psychiatry 2012;17:494-502.

19 Borovcanin M, Jovanovic I, Radosavljevic G, Djukic Dejanovic S, Bankovic D, Arsenijevic N, Lukic ML: Elevated serum level of type-2 cytokine and low IL-17 in first episode psychosis and schizophrenia in relapse. J Psychiatr Res 2012;46:1421-1426. 
20 Şimşek Ş, Ylldlrlm V, Çim A, Kaya S: Serum IL-4 and IL-10 levels correlate with the symptoms of the drug-naive adolescents with first episode, early onset schizophrenia. J Child Adolesc Psychopharmacol 2016;26:721-726.

21 Noto C, Ota VK, Santoro ML, Ortiz BB, Rizzo LB, Higuchi CH, Cordeiro Q, Belangero SI, Bressan RA, Gadelha A, Maes M, Brietzke E: Effects of depression on the cytokine profile in drug naïve first-episode psychosis. Schizophr Res 2015;164:53-58.

22 Higgins JP, Thompson SG, Deeks JJ, Altman DG: Measuring inconsistency in meta-analyses. BMJ 2003;327:557-560.
23 Borovcanin M, Jovanovic I, Radosavljevic G, Djukic Dejanovic S, Stefanovic V, Arsenijevic N, Lukic ML: Antipsychotics can modulate the cytokine profile in schizophrenia: Attenuation of the type-2 inflammatory response. Schizophr Res 2013;147:103-109.

24 Noto C, Ota VK, Gouvea ES, Rizzo LB, Spindola LM, Honda PH, Cordeiro Q, Belangero SI, Bressan RA, Gadelha A, Maes M, Brietzke $\mathrm{E}$ : Effects of risperidone on cytokine profile in drug-naive first-episode psychosis. Int J Neuropsychopharmacol 2014;18:pyu042.

25 Samoud S, Elkissi Y, Ayachi M, Hannachi N, Belhadjali B, Boukadida J: Increased interleukin-17 and decreased BAFF serum levels in acute phase of schizophrenia. Schizophr Res 2010;117:487.
26 Dimitrov DH, Lee S, Yantis J, Valdez C, Paredes RM, Braida N, Velligan D, Walss-Bass C: Differential correlations between inflammatory cytokines and psychopathology in veterans with schizophrenia: potential role for IL17 pathway. Schizophr Res 2013;151:29-35.

27 Himmerich H, Schönherr J, Fulda S, Sheldrick AJ, Bauer K, Sack U: Impact of antipsychotics on cytokine production in-vitro. J Psychiatr Res 2011;45:1358-1365.

28 Upthegrove R, Barnes NM: The immune system and schizophrenia: an update for clinicians. Adv Psychiatr Treat 2014;20:83-91.

29 Smith RS, Maes M: The macrophage-T-lymphocyte theory of schizophrenia: additional evidence. Med Hypotheses 1995;45:135-141. 